\title{
Revista Colombiana de

\section{Guía de práctica clínica para la prevención, detección temprana, diagnóstico, tratamiento y seguimiento de las dislipidemias: evaluación del riesgo cardiovascular}

\author{
Oscar M. Muñoz ${ }^{a, b}$, Ángel A. García ${ }^{a, b, *}$, Daniel Fernández-Ávila ${ }^{a, b}$, \\ Angélica Higuera $^{\mathrm{a}, \mathrm{b}}$, Álvaro J. Ruiz ${ }^{\mathrm{a}, \mathrm{b}}$, Pablo Aschner ${ }^{\mathrm{a}}$, Juan M. Toro ${ }^{\mathrm{c}, \mathrm{d}}$, \\ Juan M. Arteaga ${ }^{e}$, Alonso Merchán ${ }^{f}$, Gregorio Sánchez ${ }^{g}$ y Yadira Villalba $^{\mathrm{h}}$ \\ a Departamento de Medicina Interna, Pontificia Universidad Javeriana, Hospital San Ignacio, Bogotá, Colombia \\ b Departamento de Epidemiología Clínica y Bioestadística, Pontificia Universidad Javeriana, Hospital San Ignacio, Bogotá, \\ Colombia \\ c Universidad de Antioquia. Medellín, Colombia \\ d Hospital Pablo Tobón Uribe. Medellín, Colombia \\ e Departamento de Medicina Interna, Universidad Nacional de Colombia. Bogotá, Colombia \\ ${ }^{f}$ Fundación Clínica Abood Shaio. Bogotá, Colombia \\ g Hospital Universitario del Quindío San Juan de Dios, Armenia, Colombia \\ h Universidad de Cartagena. Cartagena, Colombia
}

Recibido el 7 de octubre de 2014; aceptado el 18 de abril de 2015

Disponible en Internet el 7 de julio de 2015

\section{PALABRAS CLAVE}

Hipercolesterolemia;

Lípidos;

Tratamiento;

Estatinas

\begin{abstract}
Resumen
Objetivo: El tratamiento de la dislipidemia debe ser acorde con el riesgo individual de cada paciente. Existen múltiples ecuaciones de predicción de riesgo cardiovascular, sin embargo, es necesario determinar cuál es la más adecuada para ser utilizada en la población colombiana. Métodos: La alianza CINETS, comisionada por el Ministerio de la Protección Social y Colciencias, revisó la evidencia disponible con respecto a qué métodos de evaluación de riesgo cardiovascular son válidos en la población colombiana. Se generaron recomendaciones utilizando la metodología GRADE.

Población: Población adulta con diagnóstico de dislipidemia o en riesgo de desarrollarla.

Recomendaciones: En la población de prevención primaria o sin enfermedad cardiovascular clínicamente manifiesta se recomienda utilizar la escala de Framingham recalibrada para Colombia
\end{abstract}

\footnotetext{
* Autor para correspondencia.

Correo electrónico: angel.garcia@javeriana.edu.co (Á.A. García).
} 
para clasificar el riesgo (Recomendación fuerte a favor de la intervención). Existen personas con condiciones que por sí mismas implican un riesgo mayor y ameritan manejo farmacológico directo.

(C) 2015 Sociedad Colombiana de Cardiología y Cirugía Cardiovascular. Publicado por Elsevier España, S.L.U. Este es un artículo Open Access bajo la licencia CC BY-NC-ND (http:// creativecommons.org/licenses/by-nc-nd/4.0/).

\section{KEYWORDS}

Hypercholesterole-

mia;

Lipid;

Treatment;

Statins
Clinical practice guidelines for the prevention, early detection, diagnosis, treatment and follow up of dyslipidemia: cardiovascular risk assessment

\begin{abstract}
Aim: The treatment of dyslipidemia must be related with patient's individual risk. There are multiple functions for predicting cardiovascular risk; however, it is necessary to determine the most appropriate for being used in Colombian population.

Methods: The CINETS alliance, endorsed by the Colombian Social Protection Ministry and Colciencias, reviewed the evidence available about the validity of cardiovascular risk evaluation methods in Colombian Population. Recommendations were generated using GRADE methodology.

Population: General adult population with or at risk of dyslipidemia.

Recommendations In primary prevention population, or without clinically evident cardiovascular disease, Framingham risk function recalibrated for Colombia must be used to calculate cardiovascular risk (Strong recommendation). In patients with an especial condition increasing cardiovascular risk, direct pharmacologic treatment must be used.

(C) 2015 Sociedad Colombiana de Cardiología y Cirugía Cardiovascular. Published by Elsevier España, S.L.U. This is an open access article under the CC BY-NC-ND license (http:// creativecommons.org/licenses/by-nc-nd/4.0/).
\end{abstract}

\section{Introducción}

En la práctica clínica disponemos de múltiples sistemas de estimación de riesgo cardiovascular ${ }^{1-9}$. Estos modelos matemáticos asignan diferentes valores a cada uno de los factores de mayor riesgo: el sexo, la edad, la presión arterial, el tabaquismo, la diabetes, los niveles de colesterol total, el colesterol de baja densidad ( $C L D L)$, el colesterol de alta densidad (cHDL) y los antecedentes familiares. En personas libres de la enfermedad cardiovascular clínicamente manifiesta (la prevención primaria) generan un estimado del riesgo de desarrollar la enfermedad cardiovascular (ECV) dentro de un determinado lapso de tiempo (por ejemplo, 10 años).

Los modelos de predicción más conocidos y probablemente los más utilizados son los modelos de riesgo de Framingham ${ }^{2}$, Systematic Coronary Risk Evaluation $(\mathrm{SCORE})^{3}$ y Prospective Cardiovascular Munster (PROCAM) ${ }^{5}$. Vale la pena resaltar adicionalmente el modelo propuesto recientemente por la Asociación Americana del Corazón (American Heart Association [AHA]) y el Colegio Americano de Cardiología (American College of Cardiology $[\mathrm{ACC}])^{10}$.

Si bien las ecuaciones de predicción de riesgo derivadas de estos estudios gozan de gran aceptación y se usan en nuestro medio, existe la evidencia que muestra que no se pueden aplicar directamente en todas las poblaciones ${ }^{4}$.
Resulta pues prioritario definir el modelo más adecuado para ser utilizado en la población de Colombia, buscando adicionalmente racionalizar el uso de recursos al seleccionar a los pacientes que serán candidatos al tratamiento farmacológico.

Adicionalmente existen grupos de pacientes en quienes no es necesario realizar estos cálculos, dado que presentan condiciones clínicas que por sí mismas confieren un riesgo cardiovascular y cerebrovascular aumentado. Reconocer estas poblaciones es por tanto importante, no solo para definir el inicio del manejo farmacológico, sino para definir la intensidad del mismo.

El Ministerio de Salud y Protección Social encargó a la Pontificia Universidad Javeriana y a la Alianza CINETS - conformada por las Universidades Javeriana, Nacional y Antioquia- el desarrollo de una Guía clínica sobre la prevención, detección temprana, diagnóstico, tratamiento y seguimiento de las dislipidemias en la población mayor de 18 años de edad. El resultado de ese proceso, en lo referente a la evaluación del riesgo cardiovascular y a la definición de poblaciones candidatas al manejo farmacológico, se presenta de forma resumida en el presente escrito. Cabe resaltar que en este proceso también participaron activamente la Asociación Colombiana de Medicina Interna, la Sociedad Colombiana de Cardiología, la Asociación Colombiana de Endocrinología, Diabetes y Metabolismo, la Asociación Colombiana de Fisioterapia, el Centro 
Colombiano de Nutrición Integral y el Centro Nacional de Investigación en Evidencia y Tecnologías en Salud (CINETS).

\section{Métodos}

El proceso de desarrollo de la guía se describe detalladamente en el Manual para el desarrollo de guías de práctica clínica y en la actualización de dicha metodología (disponible en la página web del Ministerio de Salud y Protección Social: http://gpc.minsalud.gov.co) ${ }^{11}$

Cabe resaltar que dicha metodología busca garantizar una búsqueda sistemática de la evidencia científica (incluyendo tanto las revisiones sistemáticas de la literatura como los estudios primarios). Así mismo, establece una metodología clara para la selección de la evidencia que se va a utilizar, evaluando cuidadosamente la calidad de la misma. La versión completa de la guía, en la que se presentan los resultados de todas estas evaluaciones, pueden ser consultadas en la página web del Ministerio de Salud y Protección Social, así como en la página web de la alianza CINETS.

El grupo metodológico preparó un resumen de la evidencia disponible y lo presentó al panel completo durante las reuniones de generación de las recomendaciones. Este panel incluyó a representantes de diversas especialidades médicas (los cardiólogos, los endocrinólogos, los médicos internistas, los médicos de familia y los médicos generales), así como a otros profesionales de la salud (los nutricionistas y los terapeutas físicos). Cabe enfatizar que durante todo el proceso se contó también con representantes de los pacientes, que aportaron activamente en la generación de las recomendaciones. Todos los integrantes del panel presentaron abiertamente la declaración de sus conflictos de interés. Estos documentos están disponibles en la versión completa de la guía.

Durante las reuniones de generación de las recomendaciones se siguió la metodología propuesta por el grupo GRADE, que permite tener en cuenta no solo la calidad de la evidencia disponible, sino las consideraciones de costos, las preferencias de los pacientes y la relación entre los beneficios y riesgos de las tecnologías de interés (las pruebas, las estrategias de manejo, las intervenciones y los medicamentos). Las recomendaciones presentadas muestran, por tanto, la fuerza de la recomendación (fuerte o débil), la dirección de la misma (a favor o en contra de la intervención), así como la calidad de la evidencia que la soporta (muy baja, baja, moderada, alta o consenso de expertos).

La versión final de la Guía fue evaluada por organismos internacionales, seleccionados por el Ministerio de la Protección Social, expertos tanto en dislipidemias como en metodología. Sus aportes fueron tenidos en cuenta por el grupo desarrollador de la Guía.

\section{Resultados}

Se condujo una búsqueda sistemática de la literatura de novo para identificar el metaanálisis o las revisiones sistemáticas de la literatura publicadas hasta mayo de 2013, empleando las bases de datos sugeridas en la guía metodológica (Medline, Cochrane, CRD Database) ${ }^{11}$.
No se identificó ningún metaanálisis ni revisión sistemática de la literatura que abordaran el tema de modelos de evaluación de riesgo cardiovascular válidos para la población colombiana. De igual forma, se realizó una búsqueda sistemática de estudios primarios (Pubmed, Embase), así como la búsqueda de la literatura gris. Solo se cuenta con un estudio ${ }^{12}$ que evalúa específicamente la validez de las escalas de predicción de riesgo en nuestro país.

En el estudio de Múñoz et al. ${ }^{12}$ se realizó la validación externa de los modelos de Framingham y PROCAM en una cohorte histórica en Bogotá, Colombia. Se evaluaron 1.013 pacientes, de entre 30 y 74 años de edad, libres de eventos cardiovasculares al ingreso a la cohorte, que se siguieron por 10 años en busca de enfermedad cardiovascular, definida como la muerte coronaria, el infarto de miocardio o la angina de pecho.

En pacientes de riesgo bajo e intermedio el número de eventos cardiovasculares predichos por la función de riesgo de Framingham sobreestimó los observados (relación entre eventos esperados y observados de 1,31 ), con una baja capacidad para separar a los pacientes de bajo riesgo de los de alto riesgo (AUC 0,65).

La función de riesgo de PROCAM ajustada por sexo tuvo una mejor capacidad de discriminación (AUC 0,74), con buena calibración (relación entre eventos esperados y observados de 1,07).

Una importante limitación del estudio fue el bajo número de pacientes de alto riesgo incluidos, por lo que no es posible generalizar los resultados de esta población. Las conclusiones de este estudio fueron que podría usarse la escala de Framingham siempre que fuera recalibrada para la población colombiana. La recalibración más adecuada resultó ser multiplicar la estimación obtenida por 0,75.

Se realizó una segunda búsqueda de la evidencia, en las mismas bases de datos, para determinar en qué grupos de pacientes resulta indicado el uso del manejo farmacológico con las estatinas, sin necesidad de realizar una cuantificación del riesgo con modelos de predicción. Se identificó la revisión sistemática de la literatura (RSL) realizada por la Cholesterol Treatment Trialists Collaboration (CTT ${ }^{13}$, que presenta información proveniente de 26 estudios clínicos que incorporaron en total a 169.138 pacientes. Cinco de esos estudios compararon 2 intervenciones diferentes basadas en las estatinas (intervenciones más intensivas frente a menos intensivas) ${ }^{14-18}$, y 21 estudios compararon manejo con las estatinas frente a placebo 0 no tratamiento ${ }^{19-39}$.

Los estudios incorporan a los pacientes con diversos factores de riesgo cardiovascular y niveles de riesgo basales. Se incluyen estudios de la prevención primaria y la prevención secundaria, así como investigaciones realizadas en pacientes con falla cardiaca, falla renal en hemodiálisis, diabetes mellitus.

Se encontró que hay una reducción de aproximadamente el $20 \%$ en el riesgo de presentar muerte de origen cardiovascular por cada $1 \mathrm{mmol} / \mathrm{l}(38,7 \mathrm{mg} / \mathrm{dl})$ de disminución en los niveles de cLDL, reducción que resulta tanto clínica como estadísticamente significativa. Cabe destacar que reducciones mayores en los niveles de colesterol cLDL conllevan reducciones igualmente mayores en el nivel de riesgo. Reducir en $2 \mathrm{mmol} / \mathrm{l}$ los niveles de cLDL reduce el 
riesgo aproximadamente en el $40 \%$, y disminuirlo $3 \mathrm{mmol} / \mathrm{l}$, hasta en el $50 \%$.

\section{Discusión}

La presente guía ya ha sido publicada, y la presente publicación amplía y complementa los conceptos presentados previamente $^{40}$. Para el momento en que desarrollamos las recomendaciones de la guía se conoció adicionalmente la propuesta desarrollada por la AHA y por el $\mathrm{ACC}^{10}$ de utilizar nuevas ecuaciones de riesgo, derivadas de los estudios de cohortes basados en la población de Estados Unidos. Dichas ecuaciones tienen algunas ventajas. La principal de ellas es que predicen el riesgo de desarrollar eventos cardiovasculares y cerebrovasculares duros (definidos como la ocurrencia de un primer infarto de miocardio no fatal, la muerte de origen coronario, o la aparición de ataque cerebrovascular fatal o no fatal), en vez de estimar el riesgo de enfermedad coronaria total como lo hace el modelo de Framingham (es decir, incluyendo la angina inestable y omitiendo la estimación del riesgo de ataque cerebrovascular). Predecir el riesgo de desarrollar los eventos cardiovasculares y los cerebrovasculares duros es en la actualidad mucho más aceptado, dado que son los desenlaces más relevantes, tanto para los médicos como para los pacientes.

A pesar de esta ventaja potencial, en el grupo se discutió la potencial aplicabilidad y utilidad de estas nuevas ecuaciones para Colombia. La conclusión del panel es que existe una alta posibilidad de que estas escalas no sean válidas en nuestros habitantes. La razón para ello es presentada directamente por el grupo desarrollador de las nuevas ecuaciones de riesgo, quienes en su manuscrito aclaran que estas ecuaciones se desarrollaron fundamentalmente en poblaciones de norteamericanos blancos y afrodescendientes, y declaran abiertamente que la representación de otros grupos étnicos fue insuficiente. Específicamente mencionan que es esperable que las nuevas ecuaciones sobreestimen el riesgo en las poblaciones de hispanos norteamericanos y recomiendan realizar estudios de validación externa para evaluar el uso de estas nuevas ecuaciones por fuera de la población en la que fue desarrollada.

Teniendo en cuenta la información presentada, y conociendo las razones para no utilizar las nuevas ecuaciones de riesgo propuestas por el ACC/AHA en el año $2013^{10}$, se decidió recomendar el uso de la escala de Framingham modificada para Colombia. Se decidió recomendar el uso del modelo de Framingham, dado que ha tenido una amplia difusión en los últimos 12 años, hay familiaridad con su uso y ha sido la propuesta por 2 consensos de la Sociedad Colombiana de Cardiología. Dicho modelo debe, no obstante, ser recalibrado con base en los datos encontrados por el estudio de Múñoz et al. ${ }^{12}$. El ajuste consiste en multiplicar el resultado de la escala por 0,75 .

Adicionalmente, el panel de expertos decidió evaluar en qué grupos de pacientes puede ser necesario indicar el manejo farmacológico sin necesidad de realizar el cálculo de riesgo cardiovascular a través de la escala de Framingham modificada para Colombia. De acuerdo con el metaanálisis presentado, reducir el cLDL basal con las estatinas en $40 \mathrm{mg} / \mathrm{dl}$ se traduce en una reducción del riesgo relativo del
$20 \%$ en eventos cardiovasculares mayores. De igual forma, una reducción del cLDL basal aproximadamente entre 40 y $80 \mathrm{mg} / \mathrm{dl}$ se traduce en una reducción de eventos entre el 40 y el $50 \%$, respectivamente. Esta reducción es similar independientemente de los niveles de colesterol basal y del riesgo global del paciente.

Dado que la reducción relativa de riesgo se mantiene independiente de los niveles de riesgo basal, los beneficios reales del tratamiento dependerán del riesgo absoluto que tenga cada paciente de presentar eventos en los siguientes 10 años. Por ejemplo, en pacientes con un riesgo basal de hasta el $10 \%$ a 10 años, la reducción absoluta del riesgo esperada sería como máximo del $2 \%$ por cada $1,0 \mathrm{mmol} / /$ que se disminuyan los niveles de cLDL. Por tanto, el número de pacientes que sería necesario tratar (NNT) sería de 50 para evitar un evento vascular mayor. En este caso podría no ser necesario el tratamiento farmacológico, y podrán recomendarse cambios en el estilo de vida (la dieta y el ejercicio). Por el contrario, en pacientes con un riesgo basal del $40 \%$ a 10 años, el impacto del tratamiento farmacológico sería mucho mayor. En este grupo la reducción absoluta del riesgo sería del $8 \%$ por cada 1,0 mmol/l que se disminuyan los niveles de CLDL, y el NNT de 12. Para disminuciones mayores de los niveles de cLDL el impacto sería aún más importante. En estos pacientes resulta claro el beneficio del manejo farmacológico con las estatinas de alta intensidad.

Teniendo en cuenta lo anterior, entramos a considerar en qué grupos de pacientes el riesgo basal es lo suficientemente alto para justificar el manejo farmacológico, y una vez definida la necesidad del manejo farmacológico definir en qué grupos de pacientes se justifica la terapia con las estatinas de alta o moderada intensidad. Cabe aclarar que para la presente guía se consideran estatinas de alta intensidad aquellas que son capaces de disminuir en un $50 \%$ o más los valores de cLDL (la atorvastatina $80 \mathrm{mg}$ o la rosuvastatina $40 \mathrm{mg}$ ), y estatinas de moderada intensidad las que pueden reducir los niveles de cLDL entre el 30 y el $50 \%$ (la atorvastatina 10, $20 \circ 40 \mathrm{mg}$, la rosuvastatina 5, 10 o $20 \mathrm{mg}$, la pravastatina $40 \mathrm{mg}$, la simvastatina $20040 \mathrm{mg}$ y la lovastatina 20 o $40 \mathrm{mg}$ ).

El consenso del grupo desarrollador fue aceptar las recomendaciones dadas por las guías del ACC/AHA en el año $2013^{10}$, que señalan los siguientes grupos de pacientes como candidatos al manejo farmacológico, específicamente con las estatinas, sin necesidad de hacer un cálculo previo del riesgo cardiovascular a 10 años:

1. Personas con historia de eventos cardiovasculares ateroscleróticos, es decir, pacientes con historia de eventos coronarios agudos (incluyendo infarto agudo de miocardio y angina estable o inestable, ataque cerebrovascular, ataque isquémico transitorio, historia previa de revascularización coronaria o a otro nivel, o enfermedad vascular aterosclerótica de miembros inferiores).

2. Personas con niveles de $\mathrm{cLDL}>190 \mathrm{mg} / \mathrm{dl}$.

3. Diabetes mellitus.

Para las personas con diagnóstico de diabetes mellitus se consideró que valía la pena diferenciar a las que tenían un riesgo especialmente elevado, dado que ellas tendrían indicación del manejo farmacológico con las estatinas de alta intensidad. El consenso del panel fue proponer manejo 


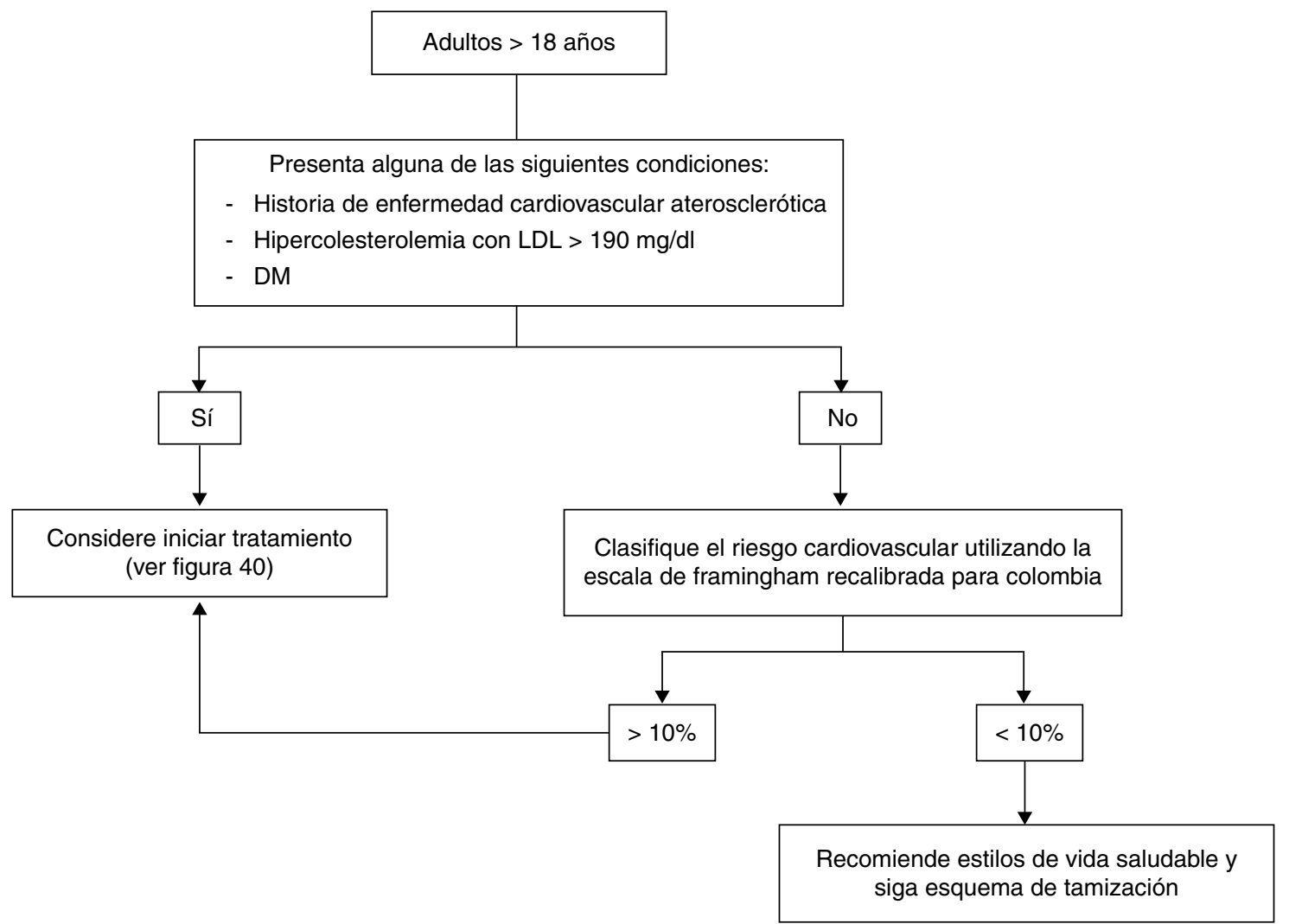

Figura 1 Recomendaciones de la guía para evaluación del riesgo cardiovascular en población colombiana mayor de 18 años.

intensivo a las personas con diabetes mellitus, mayores de 40 años de edad, que presenten asociado al menos un factor de riesgo cardiovascular asociado y niveles de $\mathrm{CLDL}>70 \mathrm{mg} / \mathrm{dl}$. Los pacientes diabéticos que no cumplan estas condiciones serán candidatos a manejo farmacológico con las estatinas de intensidad moderada.

El consenso del grupo es que en estos pacientes el costo de la intervención farmacológica es razonable (con NNT bajos) y el balance entre los riesgos y los beneficios es aceptable.

Adicionalmente se discutió en el grupo que algunas condiciones adicionales podrían ser tenidas en cuenta a la hora de definir el inicio de manejo con las estatinas de moderada intensidad. Todas ellas, de estar presentes, podrían justificar el inicio de manejo farmacológico una vez que se evalúe el balance de riesgos y beneficios, así como las preferencias individuales de los pacientes:

- Niveles de colesterol cLDL > $160 \mathrm{mg} / \mathrm{dl}$.

- Historia familiar de eventos cardiovasculares o cerebrovasculares tempranos en familiares en primer grado de consanguinidad (hombres menores de 55 años de edad o mujeres menores de 65 años de edad).

\section{Conclusiones}

A continuación se presentan las recomendaciones finales de la Guía de práctica clínica para la prevención, detección temprana, diagnóstico, tratamiento y seguimiento de las dislipidemias, en cuanto a la evaluación del riesgo cardiovascular. Cabe aclarar que las recomendaciones en cuanto al manejo farmacológico recomendado en los pacientes, según su riesgo cardiovascular, se presentan en un segundo artículo de esta serie ${ }^{41}$. Las recomendaciones fueron derivadas de la evidencia presentada, y las consideraciones mostradas en la discusión. La información completa del proceso para generar las recomendaciones se encuentra en la versión completa de la Guía, disponible a través de la página electrónica del Ministerio de Protección Social, http://gpc.minsalud.gov.co.

Esta misma información se presenta de forma gráfica a manera de algoritmo (fig. 1).

\section{Recomendación}

En la población de la prevención primaria o sin la enfermedad cardiovascular clínicamente manifiesta se recomienda utilizar la escala de Framingham recalibrada para Colombia ${ }^{a}$ para clasificar el riesgo (Recomendación fuerte a favor de la intervención).

\section{Puntos de buena práctica clínica}

- Las personas en los siguientes grupos tienen condiciones que por sí mismas implican un riesgo mayor y ameritan

\footnotetext{
a La escala de riesgo recalibrada para Colombia se entiende como el cálculo derivado de la escala original de Framingham multiplicado por 0,75 .
} 
manejo farmacológico. Por tanto, no es necesario utilizar tablas para el cálculo de riesgo:

- Historia de la enfermedad cardiovascular ateroscleróticab.

- Niveles de CLDL mayores de $190 \mathrm{mg} / \mathrm{dl}$.

- Diabetes mellitus.

- Es aconsejable que en la consulta de primera vez el médico tenga un tiempo disponible de al menos $30 \mathrm{~min}$; esto con el fin de:

- Facilitar una adecuada aplicación de las tablas de riesgo, como la propuesta por Framingham.

- Asegurar una suficiente explicación al paciente sobre el significado del riesgo coronario y el correspondiente tratamiento, que incluya cambios terapéuticos en estilo de vida y pronóstico.

\section{Responsabilidades éticas}

Protección de personas y animales. Los autores declaran que para esta investigación no se han realizado experimentos en seres humanos ni en animales.

Confidencialidad de los datos. Los autores declaran que en este artículo no aparecen datos de pacientes.

Derecho a la privacidad y consentimiento informado. Los autores declaran que en este artículo no aparecen datos de pacientes.

\section{Financiación}

Ministerio de la Protección Social-Colciencias.

\section{Conflicto de intereses}

Ninguno para la presente sección de la guía.

\section{Agradecimientos}

Agradecemos la contribución de las personas que, en carácter de representantes de expertos temáticos, usuarios, población blanco o grupos de interés, asistieron o participaron en las reuniones de socialización realizadas durante el desarrollo de la presente guía.

Agradecemos la participación de las instituciones, asociaciones y sociedades científicas, a través de sus representantes, por sus aportes y contribución en los diferentes procesos del desarrollo de la guía:

Asociación Colombiana de Medicina Interna (ACMI).

Sociedad Colombiana de Cardiología y Cirugía Cardiovascular.

Asociación Colombiana de Endocrinología, Diabetes y Metabolismo.

\footnotetext{
b Se consideran pacientes con historia de enfermedad cardiovascular aterosclerótica a los que hayan presentado eventos coronarios agudos (incluyendo el infarto agudo de miocardio y la angina estable o inestable), ataque cardiovascular, ataque isquémico transitorio, historia previa de revascularización (coronaria o a otro nivel) o enfermedad vascular aterosclerótica de miembros inferiores.
}

Asociación Colombiana de Fisioterapia (ASCOFI). Centro Colombiano de Nutrición Integral (CECNI).

Centro Nacional de Investigación en Evidencia y Tecnologías en Salud (Alianza CINETS).

\section{Bibliografía}

1. Wilson PW, D’Agostino RB, Levy D, Belanger AM, Silbershatz H, Kannel WB. Prediction of coronary heart disease using risk factor categories. Circulation. 1998;97:1837-47.

2. D'Agostino RB, Vasan RS, Pencina MJ, Wolf PA, Cobain M, Massaro JM, et al. General cardiovascular risk profile for use in primary care: The Framingham Heart Study. Circulation. 2008;117:743-53.

3. Conroy RM, Pyörälä K, Fitzgerald AP, Sans S, Menotti A, De Backer G, et al. Estimation of ten-year risk of fatal cardiovascular disease in Europe: The SCORE project. Eur Heart J. 2003;24:987-1003.

4. Woodward M, Brindle P, Tunstall-Pedoe H. Adding social deprivation and family history to cardiovascular risk assessment: The ASSIGN score from the Scottish Heart Health Extended Cohort (SHHEC). Heart. 2007;93:172-6.

5. Assmann G. Simple scoring scheme for calculating the risk of acute coronary events based on the 10-year follow-up of the prospective cardiovascular munster (PROCAM) study. Circulation. 2002;105:310-5.

6. World Health Organization (WHO). Prevention of Cardiovascular Disease. Ginebra: WHO; 2007.

7. Hippisley-Cox J, Coupland C, Robson J, Brindle P. Predicting cardiovascular risk in England and Wales: Prospective derivation and validation of QRISK2. BMJ. 2008;336: 1475-82.

8. Ridker P, Paynter NP, Rifai N, Gaziano M, Cook N. C-reactive protein and parental history improve global cardiovascular risk prediction: The Reynolds Risk Score for men. Circulation. 2008; 118:2243-51.

9. Ridker PM, Buring JE, Rifai N, Cook NR. Algorithms for the assessment of global cardiovascular risk in women. JAMA. 2007;297:611-9.

10. Goff D, Lloyd-Jones DM, Bennett G, Coady S, D’Agostino RB, Gibbons R, et al. 2013 ACC/AHA Guideline on the Assessment of Cardiovascular Risk: A Report of the American College of Cardiology/American Heart Association Task Force on Practice Guidelines. Journal of the American College of Cardiology. Disponible en: http://www.ncbi.nlm.nih.gov/pubmed/24239921

11. Carrasquilla G, Pulido $A$, Mieth $K$, Múñoz $O$, de la Hoz $A M$, Guerrero R. Guía Metodológica para la elaboración de Guías de Práctica Clínica con Evaluación Económica en el Sistema General de Seguridad Social en Salud Colombiano. 2013.

12. Múñoz $\mathrm{O}$, Ruiz A, Rodríguez N. Validación de los modelos de predicción de Framingham y PROCAM como estimadores de riesgo cardiovascular en una población colombiana. Rev Colomb Cardiol. 2014;21:202-12.

13. Baigent C, Blackwell L, Emberson J, Holland LE, Reith C, Bhala $\mathrm{N}$, et al. Efficacy and safety of more intensive lowering of LDL cholesterol: A meta-analysis of data from 170,000 participants in 26 randomised trials. Lancet. 2010;376:1670-81.

14. Cannon CP, Braunwald E, McCabe CH, Rader DJ, Rouleau JL, Belder R, et al., Pravastatin or Atorvastatin Evaluation and Infection Therapy-Thrombolysis in Myocardial Infarction 22 Investigators. Intensive versus moderate lipid lowering with statins after acute coronary syndromes. New Eng J Med. 2004;350:1495-504.

15. Pedersen TR, Faergeman O, Kastelein JJ, Olsson AG, Tikkanen MJ, Holme I, et al. Simvastatin for secondary prevention. JAMA. 2013;294:2437-46. 
16. de Lemos JA, Blazing MA, Wiviott SD, Lewis EF, Fox KAA, White $\mathrm{HD}$, et al. Early intensive vs a delayed conservative simvastatin strategy in patients. JAMA. 2013;292:1307-16.

17. LaRosa JC, Grundy SM, Waters DD, Shear C, Barter P, Fruchart JC, et al. Intensive lipid lowering with atorvastatin in patients with stable coronary disease. New Eng J Med. 2005;352:1425-35.

18. Armitage J, Bowman L, Wallendszus K, Bulbulia R, Rahimi K, Haynes R, et al. Intensive lowering of LDL cholesterol with $80 \mathrm{mg}$ versus $20 \mathrm{mg}$ simvastatin daily in 12,064 survivors of myocardial infarction: A double-blind randomised trial. Lancet. 2010;376:1658-69.

19. Downs R, Clearfield M. Primary prevention of acute coronary events with lovastatin in men and women with average cholesterol levels: Results of AFCAPS/TexCAPS. JAMA. 1998;279:1615-22.

20. GISSI Prevenzione Investigators (Gruppo Italiano per lo Studio della Sopravvivenza nell'Infarto Miocardico). Results of the low dose $(20 \mathrm{mg}$ ) pravastatin GISSI Prevenzione trial in 4271 patients with recent myocardial infarction: Do stopped trials contribute to overall knowledge? Ital Heart J. 2000;1:810-20.

21. Serruys $P$, de Feyter $P$, Macaya $C$. Fluvastatin for prevention of cardiac events following successful first percutaneous coronary intervention. JAMA. 2002;287:3215-22.

22. ALLHAT Officers and Coordinators for the ALLHAT Collaborative Research Group. The Antihypertensive and LipidLowering Treatment to Prevent Heart Attack Trial. Major outcomes in moderately hypercholesterolemic, hypertensive patients randomized to pravastatin vs usual care. JAMA. 2002;298:2998-3007.

23. Scandinavian Simvastatin Survival Study Group. Randomised trial of cholesterol lowering in 4444 patients with coronary heart disease: The Scandinavian Simvastatin Survival Study (4S). Lancet. 1994;344:1383-9.

24. Holdaas H, Fellström B, Jardine AG, Holme I, Nyberg G, Fauchald $P$, et al. Effect of fluvastatin on cardiac outcomes in renal transplant recipients: A multicentre, randomised, placebocontrolled trial. Lancet. 2003;361:2024-31.

25. Koren MJ, Hunninghake DB. Clinical outcomes in managed-care patients with coronary heart disease treated aggressively in lipid-lowering disease management clinics: The alliance study. J Am Coll Cardiol. 2004;44:1772-9.

26. Sever PS, Dahlöf B, Poulter NR, Wedel H, Beevers G, Caulfield M, et al. Prevention of coronary and stroke events with atorvastatin in hypertensive patients who have average or lower-thanaverage cholesterol concentrations, in the Anglo-Scandinavian Cardiac Outcomes Trial-Lipid Lowering Arm (ASCOT-LLA): A multicentre randomised controlled trial. Drugs. 2004;64 Suppl 2:43-60.

27. Knopp RH, d'Emden M, Smilde JG, Pocock SJ. Efficacy and safety of atorvastatin in the prevention of cardiovascular end points in subjects with type 2 diabetes: The Atorvastatin Study for Prevention of Coronary Heart Disease Endpoints in non-insulin-dependent diabetes mellitus (ASPEN). Diabetes Care. 2006;29:1478-85.

28. Fellström BC, Jardine AG, Schmieder RE, Holdaas H, Bannister K, Beutler J, et al. Rosuvastatin and cardiovascular events in patients undergoing hemodialysis. New Eng $\mathrm{J}$ Med. 2009;360:1395-407.
29. Colhoun HM, Betteridge DJ, Durrington PN, Hitman GA, Neil HA, Livingstone SJ, et al. Primary prevention of cardiovascular disease with atorvastatin in type 2 diabetes in the Collaborative Atorvastatin Diabetes Study (CARDS): Multicentre randomised placebo-controlled trial. Lancet. 2004;364: 685-96.

30. Sacks F, Pfeffer M, Moye L, Rouleau JL, Rutherford JD, Cole TG, et al. The effect of pravastatin on coronary events after myocardial infarction in patients with average cholesterol levels. Cholesterol and Recurrent Events Trial investigators. New Eng J Med. 1996;335:1001-9.

31. Wanner C, Krane V, März W, Olschewski M, Mann JF, Ruf G, et al. Atorvastatin in patients with type 2 diabetes mellitus undergoing hemodialysis. New Eng J Med. 2005;353: 238-48.

32. Gissi-HF Investigators, Tavazzi L, Maggioni AP, Marchioli R, Barlera S, Franzosi MG, et al. Effect of rosuvastatin in patients with chronic heart failure (the GISSI-HF trial): A randomised, double-blind, placebo-controlled trial. Lancet. 2008;372: 1231-9.

33. Heart Protection Study Collaborative Group. MRC/BHF Heart Protection Study of cholesterol lowering with simvastatin in 20536 high-risk individuals: A randomised placebo-controlled trial. Lancet. 2002;360:7-22.

34. Ridker PM, Danielson E, Fonseca FA, Genest J, Gotto AM Jr, Kastelein $\mathrm{JJ}$, et al. Rosuvastatin to prevent vascular events in men and women with elevated C-reactive protein. New Eng J Med. 2008;219:5-207.

35. The Long-Term Intervention with Pravastatin in Ischaemic Disease (LIPID) Study Group. Prevention of cardiovascular events and death with pravastatin in patients with coronary heart disease and a broad range of initial cholesterol levels. New Eng J Med. 1998;339:1349-57.

36. Nakamura H, Arakawa K, Itakura H, Kitabatake A, Goto Y, Toyota $\mathrm{T}$, et al. Primary prevention of cardiovascular disease with pravastatin in Japan (MEGA Study): A prospective randomised controlled trial. Lancet. 2006;368:1155-63.

37. Post Coronary Artery Bypass Graft Trial Investigators. The effect of aggressive lowering of low-density lipoprotein cholesterol levels and low-dose anticoagulation on obstructive changes in saphenous-vein coronary-artery bypass grafts. New Eng J Med. 1997;336:153-62.

38. Shepherd J, Blauw GJ, Murphy MB, Bollen EL, Buckley BM, Cobbe $S M$, et al. Pravastatin in elderly individuals at risk of vascular disease (PROSPER): A randomised controlled trial. Lancet. 2002;360:1623-30.

39. Davis DR. Prevention of coronary heart disease with pravastatin. New Eng J Med. 1996;334:1334.

40. Muñoz O, García A, Fernández D, Higuera A, Ruiz Á, Aschner P. Guía de práctica clínica para la prevención, detección temprana, diagnóstico, tratamiento y seguimiento de las dislipidemias en la población mayor de 18 años. Acta Médica Colomb. 2014;39(S 2):1-27.

41. Muñoz OM, García A, Fernández D, Higuera A, Ruiz AJ, Aschner $P$, et al. Guía de práctica clínica para la prevención, detección temprana, diagnóstico, tratamiento y seguimiento de las dislipidemias: tratamiento farmacológico con las estatinas. Rev Colomb Cardiol. 2015. Disponible en: http://dx.doi.org/10.1016/j.rccar.2015.02. 001. 